\title{
Nutzerforschung/Benutzerforschung
}

Marvin Hubert, Joachim Griesbaum und Christa Womser-Hacker

\section{Usability von Browsererweiterungen zum Schutz vor Tracking}

\author{
https://doi.org/10.1515/iwp-2020-2075
}

\section{Demographischer Fragebogen}

Erhebung der demographischen Daten der Probanden zum Abschluss einer jeden Sitzung.

1. Welchem Geschlecht gehören Sie an? (weiblich | männlich | divers)

2. Welcher Altersgruppe gehören Sie an? (18-24 | 25-35 | 36-45 | 46-55 | >55)

3. Was ist Ihr höchster Bildungsabschluss?

(kein Abschluss | Hauptschulabschluss | Realschulabschluss | Abitur/Fachabitur | Hochschulabschluss)

4. Bitte geben Sie Ihren momentanen beruflichen Status an. (angestellt | selbstständig | arbeitssuchend | Auszubildende/r | Bachelorstudent/in | Masterstudent/in |

Doktorand/in | Rentner/in)

5. Wie viele Stunden verbringen Sie durchschnittlich täglich im Internet? (weniger als 1 Stunde | 1-3 Stunden | 4-6 Stunden | 7-9 Stunden | mehr als 9 Stunden)

6. Haben Sie einen informationstechnischen (IT-) Hintergrund und/oder verfügen Sie über Kenntnisse der Webentwicklung? (ja | nein)

7. Wie schätzen Sie Ihre EDV- bzw. Computerkenntnisse ein? ( $1=$ keine Kenntnisse, 5 = sehr gute Kenntnisse) 